\title{
The Role of C Fibers in Spinal Microglia Induction and Possible Relation with TRPV3 Expression During Chronic Inflammatory Arthritis in Rats
}

\author{
Sasan Gazerani ${ }^{1}$, Jalal Zaringhalam ${ }^{1,2^{*}}$, Homa Manaheji ${ }^{1,2}$, Sahar Golabi ${ }^{1}$ \\ 1. Department of Physiology, Faculty of Medicine, Shahid Beheshti University of Medical Sciences, Tehran, Iran \\ 2. Neurophysiology Research Center, Shahid Beheshti University of Medical Sciences, Tehran, Iran.
}

Crtation: Gazerani, S., Zaringhalam, J., Manaheji, H., \& Golabi, S. (2016). The role of C fibers in spinal microglia induction and possible relation with TRPV3 expression during chronic inflammatory Arthritis in rats. Basic and Clinical Neuroscience, 7(4), 231-240. http://dx.doi.org/10.15412/J.BCN.03070308

: http://dx.doi.org/10.15412/J.BCN.03070308

Article info:

Received: 02 March 2015

First Revision: 28 March 2015

Accepted: 27 August 2015
Key Words:

Hyperalgesia, TRPV3,

Microglia, Fractalkine

\begin{abstract}
A B S T RA C T
Introduction: Stimulation of peptidergic fibers activates microglia in the dorsal horn. Microglia activation causes fractalkine (FKN) release, a neuron-glia signal, which enhances pain. The transient vanilloid receptor 1 (TRPV1) mediates the release of neuropeptides, which can subsequently activate glia. TRPV1 and TRPV2 are generally expressed on C and A $\delta$ fibers, respectively. Expression of both proteins is upregulated during inflammation, but expression of TRPV3 after induction of inflammation is unclear.
\end{abstract}

Methods: Adult male Wistar rats were used in all experiments. Arthritis was induced in them by single subcutaneous injection of complete Freund's adjuvant (CFA) in their right hindpaws. Resiniferatoxin (RTX) was used to eliminate peptidergic fibers. We examined the relation between FKN and TRPV3 expression by administration of anti-FKN antibody.

Results: Our study findings indicated that 1) spinal TRPV3 was mostly expressed on nonpeptidergic fibers, 2) expression of spinal TRPV3 increased following inflammation, 3) elimination of peptidergic fibers decreased spinal TRPV3 expression, 4) alteration of hyperalgesia was compatible with TRPV3 changes in RTX-treated rat, and 5) anti-FKN antibody reduced spinal TRPV3 expression.

Discussion: It seems that the hyperalgesia variation during different phases of CFA-induced arthritis correlates with spinal TRPV3 expression variation on peptidergic fibers. Moreover, spinal microglial activation during CFA inflammation is involved in TRPV3 expression changes via FKN signaling.

\section{Introduction}

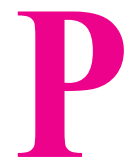

ain is described as "an unpleasant" sensory and emotional felling accompanied with different aspects of tissue injuries (Fernandez-Carvajal, Fernandez-Ballester, Devesa,
Gonzalez-Ros, \& Ferrer-Montiel, 2012; Fein, 2012). The inflammatory mediators activate the ends of pain nerve processes. Excitation of pain receptors stimulates the specialized nerves, such as $\mathrm{C}$ and $\mathrm{A} \delta$ fibers that carry pain impulses to the spinal cord (Omoigui, 2007). C and A $\delta$ fibers are essential for determining painful stimuli and launching pain sensation

* Corresponding Author:

Jalal Zaringhalam, $M D, P h D$

Address: Department of Physiology, Neurophysiology Research Center, Shahid Beheshti University of Medical Sciences, Tehran, Iran

Tel: +98 (912) $5168313 \quad$ Fax: +98 (21) 22439971

E-mail:jzaringhalam@yahoo.com 
(Chen \& Pan, 2006). Some authors suggested that sharp pain is transmitted by A $\delta$ fibers that carry information from damaged thermal and mechanical nociceptors, and it is followed by a more prolonged aching pain which is transmitted by $\mathrm{C}$ fibers that carry signals from polymodal nociceptors (Kandel, Schwartz, Jessel, Siegelbaum, \& Hudspeth, 2013).

The nociceptors are equipped with a wide range of receptors that render them sensitive to thermal, mechanical, and some chemical stimuli. Transient receptor potential (TRP) channels are temperature sensitive and when activated, depolarize nociceptor terminals. Thermo TRP channels in mammals are TRPV, TRPM, and TRPA subfamilies. Expression of TRP receptors on related nerve fibers vary during different physiological and pathological situations. Transient vanilloid receptor 1 (TRPV1) receptors are activated by capsaicin, heat, and low pH (Spicarova \& Palecek, 2008; Brandt et al., 2012). Mice deficient of TRPV1 shows impaired pain response to heat (Chen \& Pan, 2006). TRPV1 receptors are predominantly expressed in small sensory $\mathrm{C}$ fibers and to a lesser extent in A $\delta$ fibers (Brandt et al., 2012). TRPV1 expression increases in all 3 types of DRG (dorsal root ganglion) neurons after CFA injection (Yu et al., 2008).

In rat dorsal root ganglia, TRPV2 is expressed primarily in myelinated afferents, most likely in A $\delta$ nociceptors (Shimosato et al., 2005). TRPV2 has a higher temperature activation threshold, above $52^{\circ} \mathrm{C}$ (Sachdeva, Pandit, \& Bafna, 2012). Moreover, previous studies indicated that TRPV2 immunoreactivity in DRG neurons is augmented 2 days after CFAinduced inflammation (Fernandez-Carvajal, et al., 2012). TRPV3 has a unique heat threshold. It is activated at innocuous temperatures with an activation threshold around $33^{\circ} \mathrm{C}$ to $35^{\circ} \mathrm{C}$ and exhibits increasing responses at higher noxious temperatures. The responsiveness of TRPV3 receptors continues to increase as the temperature rises in noxious range. These receptors can also sensitize, suggesting that TRPV3 receptors may contribute in pain sensitization (Sachdeva, Pandit, \& Bafna, 2012). Although some studies demonstrated that acute noxious heat thermo-sensation is diminished in TRPV3-/- mice, there is no evidence about the role of TRPV3 receptors in hyperalgesia changes after inflammatory insult (Fernandez-Carvajal, et al., 2012).

More studies about pathological pain have primarily focused around neuronal mechanisms involved in pathological pain; however, neighboring astrocytes and microglia have been recognized as a powerful modulator of pain too (Milligan, Watkins, 2009). Microglia are further activated after various insults such as nerve injury, by displaying morphological changes and upregulation of microglia markers such as Ibal (Ji, Berta, \& Nedergaard, 2013). The expression of Ibal is also upregulated in activated microglia following facial nerve axotomy, inflammatory condition, and viral infections (Patro, Nagayach, \& Patro, 2010). Activated glia releases various substances such as proinflammatory cytokines and other mediators, which can enhance pain transmission (Shan et al., 2007).

Studies also indicated that proinflammatory agents such as nerve growth factor (NGF), bradykinin, and chemokines lead to TRPV1 sensitization (Palazzo et al., 2010). Fractalkine $(\mathrm{FKN})$ is a chemokine expressed on the extracellular surface of spinal neurons and sensory afferents (Milligan et al., 2004). It is a critical mediator of spinal neuron-microglial communication in chronic pain. Previous studies indicated that membrane bound FKN is located on neurons in the dorsal horn of the spinal cord (Staniland et al., 2010), whereas its receptor is exclusive and primarily expressed by microglia (Milligan et al., 2004; Staniland et al., 2010). FKN protein increases in (CX3CR1) KO mice following inflammation, and loss of FKN/receptor attenuates hyperalgesia (Staniland et al., 2010).

Altogether, we aimed to determine the role of $\mathrm{C}$ fibers in hyperalgesia, spinal microglia induction, and its possible relation with TRPV3 expression during chronic inflammatory arthritis in male rats.

\section{Material and Methods}

\subsection{Laboratory animals}

In this study adult male Wistar rats are used in weighing 180-220 gr. They were protected in Plexiglas cages under hygienic and standard condition such as 12/12-hour light/ dark cycle and food and water ad libitum. All experiments achieved the local and international standards for research on experimental animals and pain induction (Zimmermann, 1983).

\subsection{Induction of adjuvant arthritis}

According to our previous studies we induced adjuvant arthritis by injection of CFA. Briefly, we injected subcutaneously $100 \mu \mathrm{L}$ of CFA (sigma, St. Louis, MO/USA) into the rats' right hindpaws. It is clear that, CFA is heat killed Mycobacterium tuberculosis suspended in sterile mineral oil $(10 \mathrm{mg} / \mathrm{mL})$. In control group we had tried only $100 \mu \mathrm{L}$ of mineral oil into same paw. Ipsilateral inflammation was seen on first day following CFA injection. According to our previous studies, first week after CFA injection considered as "inflammatory phase" and the next two week named as arthritic phase (Tekieh et al., 2011). Then, all assessments were done on days $0,7,14$, and 21 after induction of arthritis 


\subsection{Thermal hyperalgesia assessment}

In order to evaluate paw withdrawal latency (PWL) following painful heat as a thermal hyperalgesia indicator, we used plantar test according to our previous study (Ugo Basile, Verse, Italy) (Zaringhalam, Tekieh, Manaheji, Akhtari, 2013). Briefly, 10 to 15 minutes prior to experiment, rats were placed in plexiglass cages to get accustomed to the new environment. Plantar test device was equipped with a digital timer for recording PWL automatically. If after 20 seconds of heating of hindpaw, the rat did not withdraw its paw, the test is interrupted to prevent further injury to animal and this time (20 seconds) is considered as cut-off time. PWL was assessed at least three times for each hindpaw in 5-10 minutes interval, and the mean was computed. The mean value of right hindpaw (the injected paw) was subtracted from the left one and the remainder considered as the "hyperalgesia sign" in painful hindpaw.

\subsection{Experimental procedures}

Rats were randomly divided into different experimental groups ( $\mathrm{n}=6$ for each group). To determine the effect of resiniferatoxin (RTX) on thermal hyperalgesia, a base assessment was performed by heat radiant and then weekly assessment was done in 5 weeks to find the maximum effect of RTX on thermal hyperalgesia. At the maximum effect of RTX, CFA injection was done to the right hindpaw (showed as an RTX+CFA group) to evaluate the effect of inflammation in C-fiber-deleted rats on days 0, 7, 14 and 21 following CFA injection. Dimethyl sulfoxide (DMSO) as diluents of RTX was used in control groups (showed as a DMSO+CFA group) and the assessments were fully performed as the RTX+CFA groups.

\subsection{Drugs}

An ultrapotent analog of capsaicin that is extracted from euphorbia resinifera, resiniferatoxin (RTX), which binds to the capsaicin receptor or TRPV1. Systemic injection of RTX could diminish C-fibers that are sensitive to RTX (Pan, 2003). DMSO was used to dissolve RTX according to manufacturer protocol. Rats in control group $(\mathrm{n}=6)$ received an equal amount of vehicle (DMSO). Rats in RTX group $(\mathrm{n}=6)$ were injected by $200 \mu \mathrm{g} / \mathrm{kg}$ of RTX (Abcam ab120339) intraperitoneally. Thermal and mechanical stimuli sensitivity of each rat was assessed before RTX or vehicle treatment from day zero.

To further evaluate the role of fractalkine in TRPV3 expression, rats were treated with anti-fractalkine neutralizing antibody. The antibody was obtained from Abcam/UK (ab7200). It was produced in rabbits immunised with recom- binant and natural rat fractalkine. According to the paper, we administered $1 \mu \mathrm{g} / \mathrm{d}$ of anti-fractalkine antibody for 7 days beginning on day 11 postinduction (Clark, Grist, Al-Kashi, Perretti, \& Malcangio, 2012). Antibody was dissolved in sterile phosphate-buffer (PBS) for intrathecal injection, and control animals received PBS. This solution was prepared fresh at most 30 minutes prior to injection.

\subsection{Lumbar spinal cord TRPV3/Iba1 detection by western blot}

After behavioral tests, western blot was used to examine the variation of TRPV3/Iba1 expression in the spinal cord. Rats were killed under ketamine/xylazine anesthesia, and their spinal cord was rapidly removed on ice and homogenized (Brinkman polytron homogenizer, $20000 \mathrm{rpm}$ for $30 \mathrm{~s}$ ) in extraction buffer, containing a cocktail of protease inhibitors. Then, it was centrifuged at $13000 \mathrm{rpm}\left(4^{\circ} \mathrm{C}\right)$ for 15 minutes. The supernatant was removed for analysis and protein concentration was determined (Bradford, 1976). Equal amounts of protein $(60 \mu \mathrm{g})$ were diluted with loading buffer. After boiling for 5 minutes, an aliquot of the diluted sample $(12 \mu \mathrm{L})$ was loaded on polyacrylamide gels. A sample from each spinal cord was loaded in each lane. Proteins were transferred to immobilon-P PVDF membranes. Nonspecific binding sites on the membrane were blocked by incubation with blocking buffer, followed by incubation with primary antibody in blocking buffer at a concentration of 1/2000. Membranes were washed 3 times with TBST (Tris-buffered saline with Tween 20) buffer and then incubated with secondary antibody in blocking buffer (Anti-rabbit IgG/abcam, 1/10000). The membranes were then incubated in stripping buffer and reprobed with beta-actin primary antibody (1:5000; Cell Signaling) as a loading control. Band intensity was measured by densitometry using image $\mathrm{J}$ version1.41, and expressed as the ratio of the intensity of the TRPV3/Ibal band to that of $\beta$-actin to account for any differences in starting TRPV3/ Iba1 proteins. Each experiment was replicated 3 times with new groups of rats.

\subsection{Statistical analysis}

Data were entered into SPSS version 18 and presented as means \pm SEM. The Student unpaired t-test was used to compare between group differences for hyperalgesia, allodynia, paw volume, and molecular studies. Descriptive 1-way analysis of variance (ANOVA) and paired t-test was used to compare PWL, PWT, paw volume, and molecular studies at given time points. Descriptive 1-way ANOVA followed by post hoc Tukey test was used to compare more than 2 groups. A P-value of $<0.05$ was considered as statistically significant. 


\section{Results}

3.1. Hyperalgesia variation during different stages of the study

RTX treatment caused a significant increase in thermal hyperalgesia in both paws in comparison to day 0 , five weeks after treatment $(\mathrm{P}<0.05)$. There were no significant differences in hyperalgesia during different stages of study in the control group (Figure 1).

CFA injection caused a significant increase in thermal hyperalgesia in right hindpaw during different stages of study. Hyperalgesia significantly increased on days 7, 14, and 21 after CFA injection compared to day 0 ( $\mathrm{P}<0.001$, for all 3 days). Paw withdrawal measurements in CFA group indicated that hyperalgesia significantly increased on day 14 compared to days 7 and 21 ( $P<0.05$, for both days, data not shown). There were no significant differences in hyperalgesia during different stages of study in the control group (Figure 2).

In RTX + CFA group, CFA injection into the right hindpaw caused a significant increase in thermal hyperalgesia on days 7,14 , and 21 compared to day 0 (5 weeks after RTX treatment was considered as day 0 for CFA injection ) $(\mathrm{P}<0.001$, $\mathrm{P}<0.01, \mathrm{P}<0.05$, respectively). Thermal hyperalgesia significantly increased on day 7 compared to days 14 and 21 $(\mathrm{P}<0.05$ for both days, data not shown) (Figure 2).

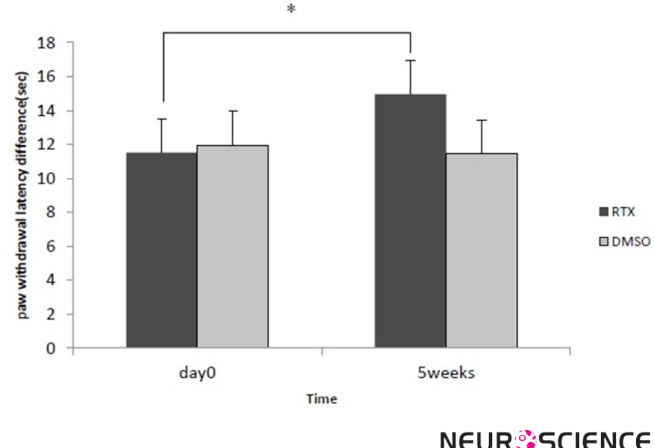

Figure 1. The effect of RTX on PWL. Data are presented as mean \pm SEM ( $n=6 /$ group). ${ }^{*}<0.05$ indicates a significant increase in PWL, 5 weeks after RTX treatment.

Thermal hyperalgesia variations in the DMSO+CFA group did not show any significant differences compared to CFA group (data not shown)

Our study showed significant difference in thermal hyperalgesia on day 7 and 14 between CFA and RTX+CFA group ( $\mathrm{P}<0.05$ for both days). There was no significant difference on day 21 between two groups (Figure 2).

3.2. Changes in spinal TRPV3 expression during different adjuvant arthritis stages

Western blot analysis of TRPV3 protein expression showed similar bands with molecular masses of approximately 90 $\mathrm{kDa}$ in tissues from the spinal cords of all experimental group rats. This finding was consistent with antibody manufacturer

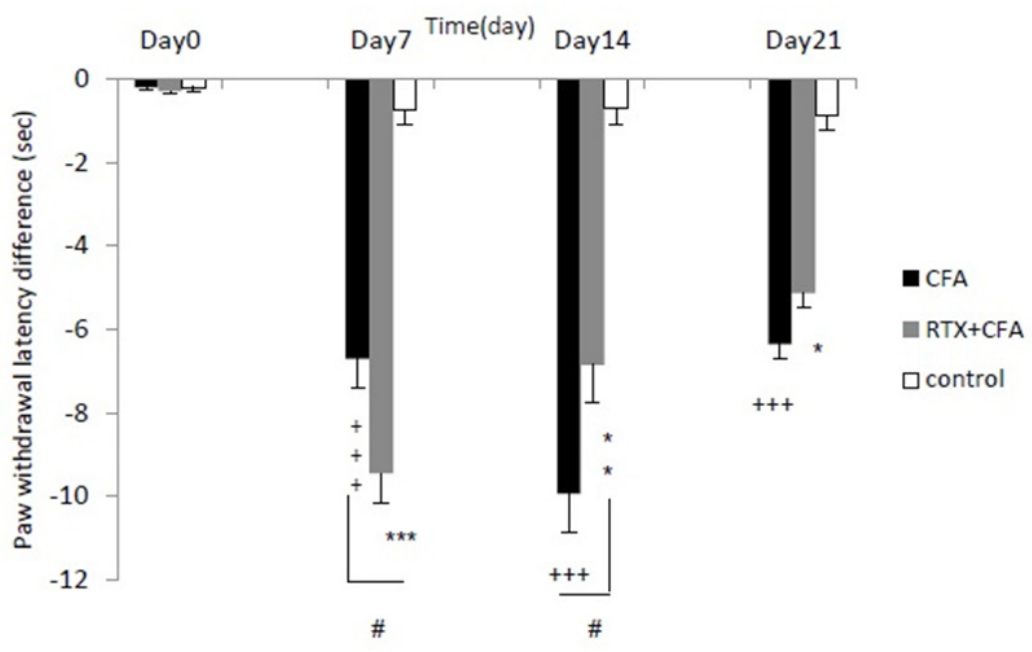

NEUR:SCIENCE

Figure 2. CFA injection into the right hindpaw causes significant hyperalgesia on days 7,14 , and 21 compared to day 0 in CFA and RTX+CFA groups. Data are presented as mean \pm SEM ( $n=6$ /group). ${ }^{*} \mathrm{P}<0.05,{ }^{* *} \mathrm{P}<0.01$, and ${ }^{* * *} \mathrm{P}<0.001$ indicates significant differences between days 7,14 , and 21 compared to day 0 in RTX+CFA group regarding hyperalgesia. $+++\mathrm{P}<0.001$ indicates significant difference between days 7, 14, and 21 compared to day 0 in CFA group regarding hyperalgesia . \# $\mathrm{P}<0.05$ indicates significant difference between RTX+CFA and CFA groups on days 7 and 14 . 
A

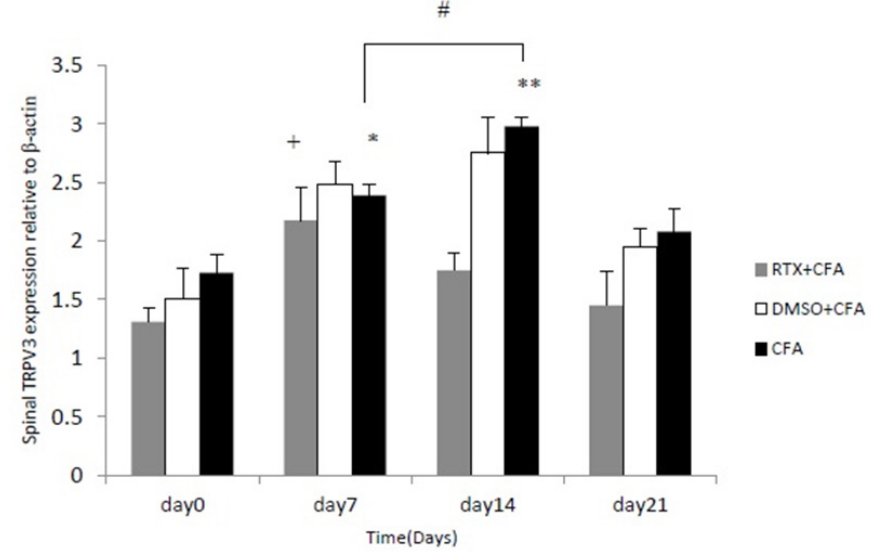

B

Day

$\begin{array}{lll}0 & 7 & 14\end{array}$

21

CFA

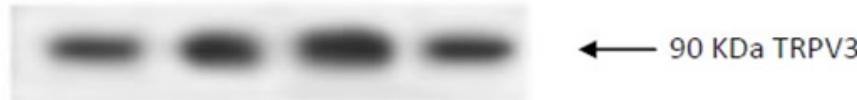

RTX

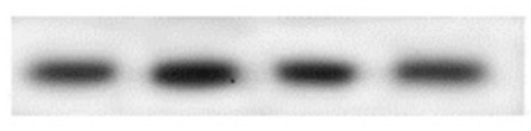

$\longleftarrow 90$ KDa TRPV3

$\mathrm{DMSO}+\mathrm{CFA}$

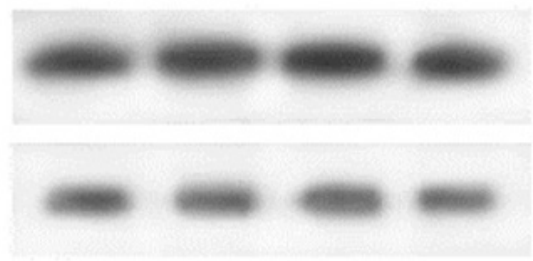

$\longleftarrow 90$ KDa TRPV3

$\beta$-actin

$\longleftarrow \quad 43 \mathrm{KDa}$

NEUR:SCIENCE

Figure 3. A) Spinal expression of TRPV3 significantly increased following CFA injection into right hindpaw. In CFA group, spinal TRPV3 expression significantly increased on days 7 and 14 compared to day 0 . In RTX+CFA group, spinal TRPV3 expression significantly increased on day 7 compared to day 0 . Data are presented as mean $\pm S E M$ ( $n=6 /$ group). ${ }^{*} P<0.05,{ }^{* *} P<0.01$ indicates significant differences between days 7 and 14 compared to day 0 in CFA group. $+\mathrm{P}<0.05$ indicates significant difference between days 7 and 0 in RTX+CFA group. \#P<0.05 indicates significant difference between days 7 and 14 in CFA group. B) Bands of western blot in CFA, RTX+CFA and DMSO+CFA groups on different days of study.

company instructions. Immunospecificity was confirmed by the absence of immunoreactive bands when the membrane was preincubated with an antigenic peptide prior to antibody incubation (data not shown). Following quantification of TRPV3-immunireactive bands, membranes were stripped and reprobed for $\beta$-actin as a loading control (43 kDa). To normalise differences in protein-loading, all data were expressed as TRPV3/ $\beta$-actin ratio. Analysis by densitometry demonstrated that CFA-induced arthritis time-dependently led to a significant increase in spinal TRPV3 protein expression compared to control group. There was a significant increase in spinal TRPV3 on days 7 and 14 compared to day 0 ( $\mathrm{P}<0.05, \mathrm{P}<0.01$, respectively). Results indicated that spinal TRPV3 expression in CFA-injected rats was significantly increased on day 14 compared to day $7(\mathrm{P}<0.05)$ (Figure 3 ).

RTX treatment did not increase TRPV3 expression 5 weeks after treatment compared to day 0 . There was also no sig- nificant change in spinal TRPV3 expression in the control group during different stages of the study (data not shown) (Figure 4).

In RTX+CFA group, CFA injection into the right hindpaw caused significant increase in spinal TRPV3 on day 7 compared day $0(\mathrm{P}<0.05)$. There were no significant differences in TRPV3 expression on days 14 and 21 compared to day 0 (Figure 3). In DMSO+CFA group, CFA injection into the right hindpaw did not show any significant differences compared to the CFA group.Our study showed significant difference between CFA and RTX+CFA group regarding TRPV3 expression on day 14 .

3.3. Effect of intrathecal anti-fractalkine inhibitory antibody on TRPV3 expression

After 1 week treatment with intrathecal administration of anti-fractalkine antibody, TRPV3 expression significantly re- 
A

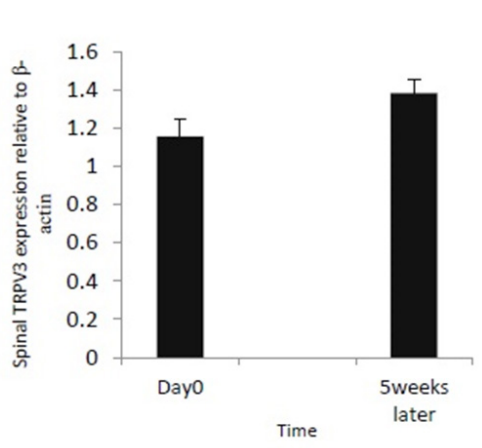

Lane1: Lane2:

B

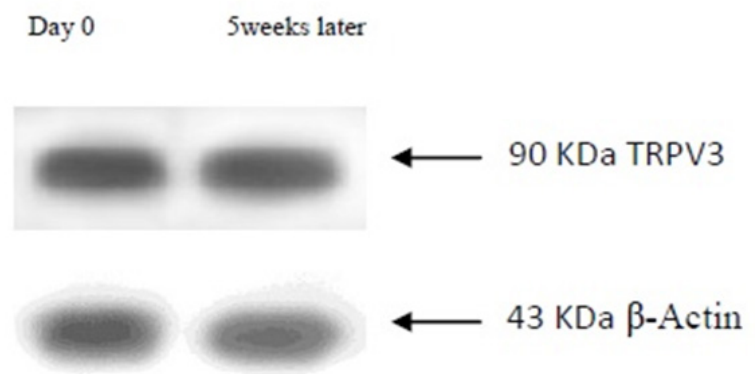

NEUR:SCIENCE

Figure 4. A) There was no significant difference in spinal TRPV3 expression 5 weeks following RTX treatment. Data are presented as mean $\pm \operatorname{SEM}(n=6 /$ group). B) lane1: day 0 before treatment, lane2: 5 weeks after RTX treatment.

A

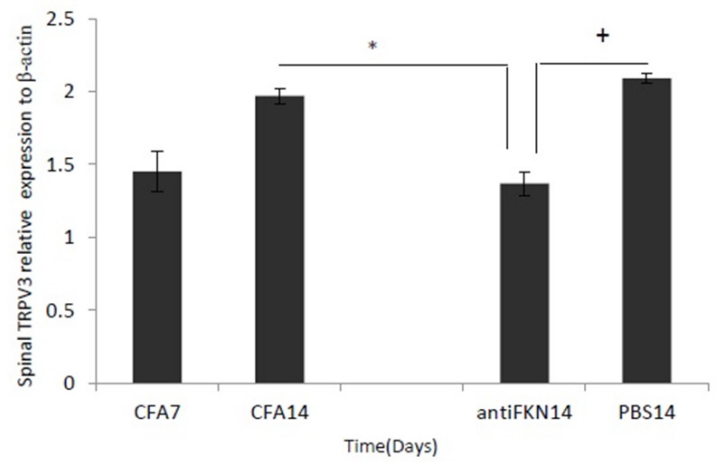

B

CFA7 CFA14 antiFKN14

TRPV3 expression in CFA and anti-fraktalkine administered group

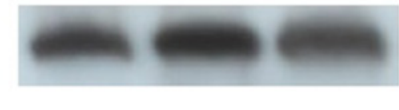

CFA7 antiFKN14 PBS14
TRPV3 expression in antifractalkine administered group and its control (PBS14)
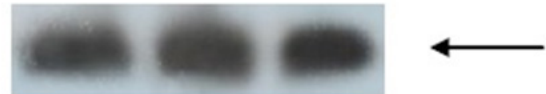

$90 \mathrm{KDa}$

$\beta$-actin

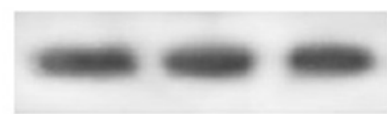

$43 \mathrm{KDa}$

NEUR:SCIENCE

Figure 5. A) Effect of 1 week anti-fractalkine receptor antibody administration on TRPV3 expression following CFA treatment (from day 7, shown as CFA7 through day 14, shown as CFA14). Data are presented as mean \pm SEM $\left(\mathrm{n}=6 /\right.$ group). ${ }^{*} \mathrm{P}<0.05$ indicates significant difference in TRPV3 expression on day 14 between CFA (CFA14) group and anti-fractalkine receptor antibody (antiFKN14) group. $+\mathrm{P}<0.05$ indicates significant difference on day 14 between anti-fractalkine receptor antibody group (antiFKN14) and the vehicle group (PBS14). B) Western blot of TRPV3 molecule. Lane1: TRPV3 expression between days 7 and 14 in CFA and anti-fractalkine receptor antibody groups. Lane2: TRPV3 expression between anti-fractalkine receptor antibody group and its vehicle group. 


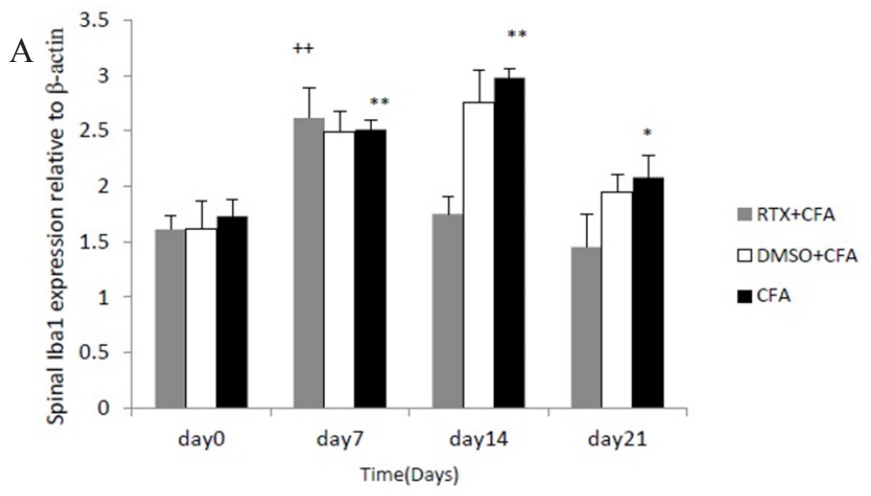

B

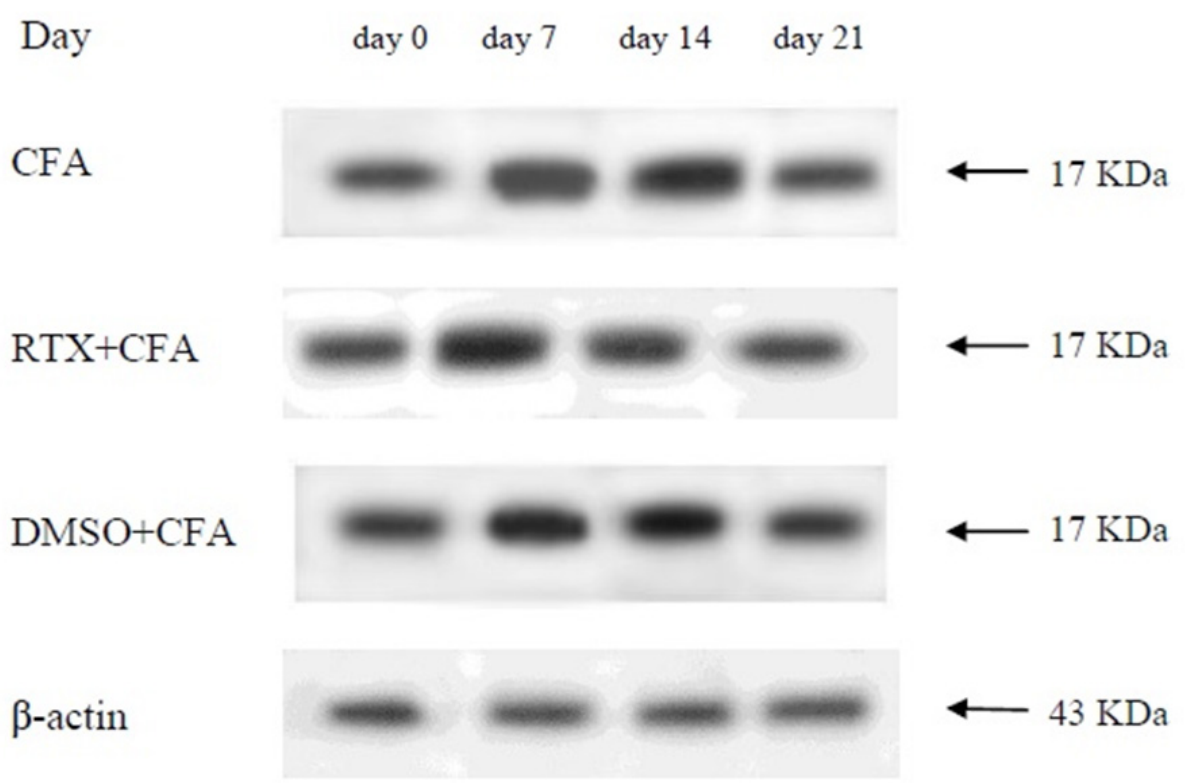

NEUR:SCIENCE

Figure 6. CFA injection into the right hindpaw caused significant alteration in Iba1 on different days of study compared to day 0. In CFA group, spinal Iba1 expression significantly increased on days 7, 14, and 21 compared to day 0 . CFA injection into the right hindpaw of previously RTX-treated rats caused significant alteration of Iba1 expression on day 7 compared to day 0 . Data are presented as mean \pm SEM $(n=6)$. ${ }^{*}<<0.05,{ }^{* *} \mathrm{P}<0.01$ indicates significant differences between days 7,14 , and $21 \mathrm{com}-$ pared to day 0 with regard to Iba1 expression in CFA group. $+\mathrm{P}<0.05$ indicates significant differences between days 7 and 0 in RTX+CFA group. There are also significant differences between two groups on day 7, 14, and 21 (data not shown). B) Bands of western blot in CFA, RTX+CFA, and DMSO+CFA groups on different study days.

duced in the spinal cord. No significant difference was seen between control group and CFA group after 1 week (Figure $5)$.

3.4. Variation in spinal Iba1 expression during different stages of adjuvant arthritis

Obtained tissue from lumbar spinal cord of all experimental groups were analyzed by western blot method for assesing Iba1 protein expression as a detector of microglia cell activity. The analysis showed similar bands with molecular weight of almost $17 \mathrm{KDa}$ which was the same as the manufacturer's instructions. To show the immunospecificity, we confirmed the absence of immunoreactive bands when we preincubated the membrane with an antigenic peptide before incubation of antibody according to our previous studies (data not shown) (Zaringhalam et al., 2013). For quantifying the Iba1-immunoreactive bands, we stripped the membranes and reprobed them for $\beta$-actin as a loading control (43 kDa). To normalise differences in protein-loading, all data were expressed as Iba1/ $\beta$-actin ratio. Analysis by densitometry demonstrated that CFA-induced arthritis time-dependently has led to significant increase in spinal Ibal protein expression compared to the control group.

CFA injection into the right hindpaw caused significant differences in Ibal expression on days 7, 14, and 21 compared 
to day 0 ( $\mathrm{P}<0.01, \mathrm{P}<0.01, \mathrm{P}<0.05$ respectively) (Figure 6). There was no significant difference in Ibal expression in the control group.

In RTX+CFA group, CFA injection into the right hindpaw caused a significant increase in Ibal expression on day 7 compared to day $0(\mathrm{P}<0.01)$. There was no significant difference in Ibal expression between days 14 and 21 compared to day 0 (Figure 6).

In DMSO+CFA group, CFA injection into the right hindpaw did not show any significant difference compared to the CFA group (data not shown).

There was a significant difference in Iba1 expression on days 7, 14, and 21 between RTX+CFA and CFA groups $(\mathrm{P}<0.05, \mathrm{P}<0.01$, and $\mathrm{P}<0.05$, respectively) (data not shown).

\section{Discussion}

Nociception is the process by which intense thermal, mechanical, or chemical stimuli are detected by peripheral nerve fibers (Basbaum et al. 2009). Peripheral nerve fibers are divided into $\mathrm{A} \delta$ and $\mathrm{C}$ fibers that are critical for detecting noxious stimuli (Chen \& Pan, 2006). Some studies suggested that primary sensory neurons are modality specific (Chen \& Pan, 2006; Hsieh, Donaldson, \& Lumb, 2015; Schmelz \& Schmidt, 2010; Pan, Khan, Alloway, \& Chen, 2003; Bahari et al., 2014). These neurons can be further divided into peptidergic and nonpeptidergic pain afferents (Ritner, Machelska, $\&$ Stein, 2009). The majority of peptidergic fibers coexpress TRPV1 receptor (Bishnoi et al., 2011), and these receptors are predominantly expressed in primary afferent fibers which are peptidergic sensory neurons (Alawi \& Keeble, 2010). Although TRPV1 is expressed in C and A $\delta$ fibers, most studies that have ablated TRPV1 fibers focus on behavioral responses associated with C fibers (McGaraughty et al., 2008; Mitchell et al., 2010; Plazzo et al., 2010). Moreover, pain signals cause enhanced nociceptive responses following glial cell induction (Shan et al., 2007; Milligan et al., 2004). Activated microglia show upregulation of Iba1, a microglia marker (Ji, Berta, \& Nedergaard, 2013; Shan et al., 2007). However, our results demonstrated that hyperalgesia, allodynia, and expression of TRPV3 and Iba1 molecule remarkably change by eliminating peripheral peptidergic fibers (mostly $\mathrm{C}$ fibers).

In the first phase of this study, our results indicated that the CFA-injected paw got involved by the onset of inflammatory criteria within several hours and continued up to day 21 . Hyperalgesia occurred on days 7, 14, and 21 following CFA treatment with its peak on day 14 that was in agreement with the results of Luo, Cheng, Han and Wan (2004) using a hot plate method with male Sprague-Dawley rats. Tekieh et al.
(2011) and Yu et al. (2008) showed that the peak reaction was on day 7; the former used heat radiant device in male Wistar rats and the latter used hot plate method in male SpragueDawley rats.

Our experiment also showed that intraperitoneal administration of RTX results in an increment of PWL during 5 weeks after treatment. As mentioned before, thermal hyperalgesia has been attributed to sensitization of myelinated $\mathrm{A} \delta$ and unmyelinated $\mathrm{C}$ primary sensory neurons (Kress, 2010). Unmyelinated nociceptors comprise the largest class of somatic afferent nerve fibers (Schmelz \& Schmidt, 2010). Based on staining methods for neuropeptides (such as Substance P and CGRP), about $50 \%$ of C fibers and $20 \%$ of A $\delta$ fibers are classified as peptidergic (Dickenson \& Kieffer, 2006). we proposed that targeting peptidergic nociceptors increased PWL about 20\% compared to day 0, most probably due to the elimination of common $\mathrm{C}$ nociceptors, which act in company with $\mathrm{A} \delta$-fibers to convey pain signals to the spinal cord (Chen \& Pan, 2006).

In the next step, CFA injection in previously RTX-treated rats (RTX+CFA group) showed a peak of hyperalgesia earlier (on day 7) in the time course. Prescot et al. (2014) demonstrated a central neuron that receives input exclusively from only one type of primary afferent neurons (PANs) with necessarily the same tuning of PAN, whereas any direct or indirect (polysynaptic) input from other PANs is liable to confer more complex tuning. Electrophysiological recordings showed spike activity of PANs with unmyelinated axons (C neurons) triggered in neurons with myelinated axons (A neurons) by stimulation of peripheral nerve or dorsal root ganglia and produce a transient depolarization in passive neighboring C neurons that share the same ganglia (Amir \& Devor, 1999).

Contrary to the classical conception, primary sensory neurons ( $\mathrm{C}$ and $\mathrm{A} \delta$ fibers) in the DRG of intact animals do not function independent from each other. There is also evidence that some previously silent DRG neurons can be cross-excited by their neighbors (Devor \& Wall, 1990). Tran, Matre and Casey (2008) demonstrated an inhibitory interaction between 2 fiber types that provide the major input to spinothalamic pathways mediating pain and temperature sensation. Altogether, we concluded that there could be many kinds of interaction (probably an inhibitory one) between PANs. Thus by omitting peptidergic fibers, interaction between $\mathrm{C}$ and $\mathrm{A} \delta$ fibers got impaired, and peak of hyperalgesia reaction occurrs earlier in the time course of the CFA model of inflammation from day 14 in CFA group to day 7 in RTX+CFA group.

Our results also indicated that TRPV3 molecule is expressed in the spinal cord. There were no changes in TRPV3 expression indicated by western blotting 5 weeks after RTX 
treatment. We inferred that nonpeptidergic fibers (mostly A $\delta$ fibers) are the main peripheral fiber which express TRPV3 molecules. In CFA group, TRPV3 expression increased on days 7 and 14 but on day 21. In this group, hyperalgesia also increased on days 7, 14, and 21, whereas in RTX+CFA group changes in TRPV3 expression was detected only on day 7; therefore, it is quite compatible with changes in hyperalgesia in RTX+CFA group. It was clear that, when temperature increases, response of TRPV3 receptors elivates, and it can consider as the sign of possible contribution of TRPV3 receptors in pain perception (Willis, 2009).Different species show different amouts of TRPV3 receptors expression among sensory neurons, then it suggests more researchs for revealing the role of TRPV3 in somatosensation. Moreover some studies revealed that, during breast pain in addition to upregulation of TRPV1 in skin cells, expression of TRPV3 is also upregulated in nociceptors (Mandadi \& Roufogalis, 2008). Smith reported TRPV3 expression and mRNA in sensory neurons (Smith et al., 2002). We presumed that in the presence of both $\mathrm{C}$ and A $\delta$ fibers, TRPV1 is the major molecule that contributes to pain transduction. However, by eliminating TRPV1-expressing fibers, TRPV3 would be the major contributor molecule in pain transduction.

Our results in the next phase of the study indicated that expression of Ibal increased in CFA group on days 7, 14, and 21 . As previously mentioned, Iba1 is a microglia activation marker. In RTX+CFA group, increment of Iba1 was only seen on day 7. Changes in Ibal expression in the RTX + CFA group was also consistent with the changes in TRPV3 expression in this group. Chen showed increased Ibal immunostaining at day 3 following capsaicin treatment (acute nociceptive model). This increment appears greater in wild type mice than TRPV1-knocked out mice (Chen,Willcockson, $\&$ Valtschanoff, 2009). Patro demonstrated that $\mathrm{Iba}^{+}$cells were prominently recorded on days 3 and 7 after which their expression was downregulated (Patro, Nagayach, \& Patro, 2010). Chen also showed that Ibal increased 7-14 days after CFA-induced arthritis. They also suggested that TRPV1-expressing sensory afferent could activate glia cells by releasing neuroactive substances. Thus, we proposed that by deleting $\mathrm{C}$ fibers which include the most peptidergic fibers, activation of microglia diminishes due to decrease in central release of neuropeptides.

In the next step, we investigated the relationship between FKN and TRPV 3 expression. Previous studies suggested that FKN (or CX3CL1) and its unique CX3CR1 receptor play a role in signaling between the neurons and microglia (Yang et al., 2012). FKN is a critical mediator of spinal neuronmicroglia communication in chronic pain (Staniland et al., 2010). Recent evidence suggested that spinal cord glia can contribute to enhanced nociceptive responses (Milligan et al., 2004), so anti-CX3CR1 neutralizing antibody attenuates thermal hyperalgesia (Milligan et al., 2004; Clark, et al., 2012). In this regard, by administrating intrathecal antiCX3CR1 antibody, expression of TRPV3 decreases in a CFA model of arthritis. It shows that expression of TRPV3 is somewhat dependent on FKN.

\section{References}

Alawi, K. H., \& Keeble, J. (2010). The paradoxical role of the transient receptor potential Vanilloid 1 receptor in inflammation. Pharmacology and Therapeutics, 125(2), 181-195.

Amir, R., \& Devor, M. (1999). Functional cross-excitation between afferent A- and C-neurons in dorsal root ganglia. Neuroscience, 95(1), 189-195.

Bahari, Z., Manaheji, H., Hosseinmardi, N., Meftahi, G. H., Sadeghi, M., Danialy, S., et al. (2014). Induction of spinal long-term synaptic potentiation is sensitive to inhibition of nNOS in L5 spinal nervetransected rats. Experimental and Clinical Sciences, 13, 751-760.

Brandt, M. R., Beyer, C. E., \& Stahl, S. M. (2012). TRPV1 Antagonists and chronic pain: Beyond thermal perception. Pharmaceuticals, 5(2), 114-132.

Basbaum, A., Bautista, D. M., Scherrer, G., \& Julius, D. (2009). Cellular and molecular mechanisms of pain. Cell, 139(2), 267-284.

Bishnoi, M., Bosgraaf, C. A., \& Premkumar, L. S. (2011). Preservation of acute pain and efferent functions following intrthecal Resiniferatoxin-induced analgesia in rats. Pain, 12(9), 991-1003.

Chen, S. R., \& Pan, H. L. (2006). Loss of TRPV1-expressing sensory neurons reduces spinal $\mathrm{Mu}$ opioid receptors but paradoxically otentiates opioid analgesia. Journal of Neurophysiology, 95(5), 30863096 .

Chen, Y., Willcockson, H. H., \& Valtschanoff, J. G. (2009). Influence of the Vanilloid receptor TRPV1 on the activation of spinal cord glia in mouse model of pain. Experimental Neurology, 220(2), 383390

Clark, A. K., Grist, J., Al-Kashi, A., Perretti, M., \& Malcangio, M. (2012). Spinal Cathepsin S and Fractalkine contribute to chronic pain in the Collagen-induced arthritis model. Arthritis $\mathcal{E}$ Rheumathism, 64(6), 2038-2047.

Devor, M., \& Wall, P. D. (1990). Cross-excitation in dorsal root ganlia of nerve-injured and intact rats. Journal of Neurophysiology, 64(60), 1733-1746.

Dickenson, A. H., \& Kieffer, B. (2006). Opiates: Basic Mechanisms. In S. B. McMahon \& M. Koltzenburg (Eds.), Wall and Melzak textbook of pain ( $5^{\text {th }}$ ed.) (pp. 3-17). London: Churchil/Livingstone.

Fernandez-Carvajal, A., Fernandez-Ballester, G., Derasa, I., Gonzales-Ros, J. M., \& Ferrer-Montiel, A. (2012). New strategies to develop novel pain therapies: Addressing thermoreceptors from diferrent points of view. Pharmaceuticals, 5(1), 16-48.

Fein, A. (2012). Nociceptors and the perception of pain (PhD thesis). Farmington: University of Connecticut Health Center. Retrieved from http://cell.uchc.edu/pdf/fein/nociceptors_fein_2012.pdf. 
Hsieh, M. T., Donaldson, L. F., \& Lumb, B. M. (2015). Differential contribution of A- and C-nociceptors to primary and secondary inflammatory hyperensitivity in the rat. Pain, 156(6), 1074-1083.

Ji, R. R., Berta, T., \& Nedergaard, M. (2013). Glia and pain: Is chronic pain a gliopathy. Pain, 154(1), 10-28.

Kandel, E. R., Schwartz, J. H. Jessel, T. M., Siegelbaum, S. A., \& Hudspeth, A. J. (2013). Principles of neural science ( $5^{\text {th }}$ ed.). New York: Mc Graw Hill.

Kress, M. (2010). Nociceptor sensitization by pro-inflammatory cytokines and chemokines. Open Pain Journal, 30(3), 453-455.

Luo, H., Cheng, J., Han, J. S., \& Wan, Y. (2004). Changes of Vanilloid receptor 1 expression in dorsal root ganglion and spinal dorsal horn duing inflammatory nociception induced by Complete Freund's adjuvant in rats. Neuroreport, 15(4), 655-658.

Milligan, E., \& Watkins, L. R. (2009). Pathological and protective roles of glia in chronic pain. Neuroscience, 10(1), 23-36.

Milligan, E. D., Zapata, V., Chacur, M., Schoeniger, D., Biedenkapp, J., O'Connor, K. A., et al. (2004). Evidence that exogenous and endogenous Fractalkin can induce spinal nociceptive facilitation in rats. European Journal of Neuroscience, 20(9), 2294-2302.

McGaraughty, S., Chu, K. L., Brown, B. S., Zhu, C. Z., Zhong, C., Joshi, S. K., et al. (2008). Contribution of central and peripheral TRPV1 receptors to mechanically evoked and spontaneous firing of spinal neurons in inflammed rats. Journal of Neurophysiology, $100(6), 3158-3166$.

Mitchell, K., Bates, B. D., Keller, J. M., Lopez, M., \& Schol, L., Navaro, J., et al. (2010). Ablation of rat TRPV1-expressing Adelta/C-fibers with Resiniferatoxin: Analysis of withdrawal behaviors, recovery of function and molecular correlates. Molecular Pain, 6, 94

Mandadi, S., \& Roufogalis, B. D. (2008). Thermo TRP channels in nociceptors: Taking a lead from capsaicin receptor TRPV1. Current Pharmacology, 6(1), 21-38.

Omoigui, S. (2007). The biochemical origin of pain- proposing a new law of pain: The origin of all pain is inflammation and the inflammatory response. Part 1 of 3- A unifying law of pain. Medical Hypothesis, 69(1), 70-82

Patro, N., Nagayach, A., \& Patro, I. K. (2010). Iba1 expression microglia in the dorsal root ganglia become activated following peripheral nerve injury in rats. Indian Journal of Experimental Biology, $48(2), 110-116$

Plazzo, E., Luongo, L., Novellis, V. D., Berrino, L., Rossi, F., \& Maione, S. (2010). moving towards supraspinal TRPV1 receptors for chronic pain. Molecular Pain, 6, 66

Prescot, S. A., Ma, Q., \& DeKonick, Y. (2014). Normal and abnormal coding of painful sensations. Natural Neuroscience, 17(2), 183-191.

Pan, H. L., Khan, G. M., Alloway, K. D., \& Chen, S. R. (2003). Resiniferatoxin induces paradoxical changes in thermal and mechanical sensitivities in rats: Mechanism of action. Journal of Neuroscience, 23(7), 2911-2919.

Ritner, H. L., Machelska, H., \& Stein, C. (2009). Immune system pain and analgesia. In I. Allan, M. Basbaum, \& C Bushnel (Eds.), Science of pain (1 ${ }^{\text {st }}$ ed.) (pp. 191-228). Baltimore: Elsevier.

Spicarova, D., \& Palecek, J. (2008). The Role of spinal cord vanilloid (TRPV1) receptors in pain modulation. Physiology Research, 57(3), 69-77.
Shimosato, G., Amaya, F., Ueda, M., Tanaka, Y., Decostered, I., \& Tanaka, M. (2005). Peripheral inflammation induces up-regulation of TRPV2 expression in rat DRG. Pain, 119(1), 225-232.

Sachdeva, T., Pandit, A., \& Bafna, P. (2012) Pain and pain receptors. International Journal of Preclinical and Pharmaceutical Research, 3(2), $82-96$

Shan, S., Hong, C., Mei, H., Ting-Ting, L., Hai-Li, P., Zhi-Qi, Z., et al. (2007). New evidence for the involvement of spinal fractalkin receptor in Pain facilitation and spinal glial activation in rat mode of monoarthritis. Pain, 129(1), 64-75.

Staniland, A. A., Clark, A. K., Wodarski, R., Sasso, O., \& Maione, T., Acquisto, F. D., et al. (2010). Reduced inflammatory and neuropathic pain and decreased spinal microglial response in Fractalkin receptor (CX3CR1) knockout mice. Journal of Neurobiochemestry, 114(4), 1143-1157.

Schmelz, M., \& Schmidt, R. (2010). Microneurographic single-unit recordings to assess receptive properties of afferent human Cfibers. Neuroscience Letters, 470(5), 158-161.

Smith, G. D., Gunthorpe, M. J., Kelsell, R. E., Hayes P. D., Reilly, P., Facer, P., et al. (2002). TRPV3 is a temperature-sensitive vanilloid receptor-like protein. Nature, 418(6894), 186-90.

Tekieh, E., Zaringhalam, J., Manaheji, H., Maghsoudi, N., Alani, B., \& Zardooz, H,. (2011). Increased serum IL-6 level time-dependently regulates hyperalgesia and spinal Mu opioid receptor expression during CFA-induced arthritis. Experimental and Clinical Science Journal, 10, 23-33.

Tran, T. D., Matre, D., \& Casey, K. (2008). An inhibitory interaction of human cortical response to stimuli preferentially exciting $\mathrm{A} \delta$ or C fibers. Neuroscience, 152(3), 798-808.

Yu, L., Yang, F., Luo, H., Liu, F. Y., Han, J. S., Xing, G. G., et al. (2008), The role of TRPV1 in different subtypes of dorsal root ganglion neurons in rat chronic inflammatory nociception induced by Complete Freund's adjuvant. Molecular Pain, 4(61), 10.

Willis, W. D. (2009). The Role of TRPV1 receptors in pain evoked by noxious thermal and chemical stimuli. Experimental Brain Research, 196(1), 5-11

Yang, J. L., Xu, B., Shuang-Shuang, L., Zhang, W. S., Xu, H., \& Deng, X. M. (2012). Gabapentin reduces CX3CL1 signaling and blocks spinal microglial activation in monoarthritic rats. Molecular Brain 5,18

Zaringhalam, J., Tekieh, E., Manaheji, H., Akhtari, Z. (2013). Cellular events during arthritis-induced hyperalgesia are mediated by Interleukin- 6 and p38 MAPK and their effects on the expression of spinal mu-opioid receptors. Rheumatology International, 33(9) 2291-2299.

Zimmermann, M. (1983). Ethical Guidelines for Investigations of Experimental Pain in Conscious Animals. Pain, 16(2), 109-110. 